\title{
Freeform Surface Characterization and Instrument Alignment for Freeform Space Applications
}

\author{
Manal Khreishi ${ }^{1,2, *}$, Raymond Ohl ${ }^{1}$, Joseph Howard ${ }^{1}$, Jonathan Papa ${ }^{1}$, Clark Hovis ${ }^{1}$, Andrew Howe ${ }^{3}$, \\ Theodore Hadjimichael ${ }^{1}$, Patrick Thompson ${ }^{1}$, Ron Shiri ${ }^{1}$, Garrett West ${ }^{1}$, Adam Phenis ${ }^{4}$, and Rongguang \\ Liang $^{2}$ \\ ${ }^{1}$ NASA Goddard Space Flight Center, 8800 Greenbelt Road, Greenbelt, MD 20771, USA \\ ${ }^{2}$ The University of Arizona, College of Optical Sciences, 1630 E University Blvd, Tucson, AZ 85721, USA \\ ${ }^{3}$ Norfolk State University CSET, 700 Park Ave., Norfolk, VA 23504, USA \\ ${ }^{4}$ AMP Optics, LLC, 13308 Midland Rd Unit 1304, Poway, CA USA \\ *manal.a.khreishi@nasa.gov
}

\begin{abstract}
CMM metrology provides simple, 3D surface data used for prescription retrieval, figure error, and alignment with high accuracy without null-correctors. Two freeform mirrors for a compact telescope were successfully characterized and aligned using the CMM.

OCIS codes: (110.0110) Imaging systems; (120.0120) Instrumentation, measurement, and metrology; (080.2208) Fabrication, tolerancing; (080.4225) Nonspherical lens design; (220.1140) Alignment; (220.4610) Optical fabrication; (220.4840) Testing; (350.1260) Astronomical optics.
\end{abstract}

\section{Introduction}

For decades, optical engineers have searched for ways to measure difficult surface prescriptions to improve the quality and reduce the cost of their fabrication, verification, and integration. State-of-the-art coordinate measuring techniques are approaching the capabilities of interferometry and, for some applications, are replacing interferometry and other optical shop techniques as the primary means of feedback to optical fabrication and requirements verification. While the hardware tools used in this work are commercially available, they are uncommon (i.e., only a few institutions use them). We discuss their capability, measurements, and data reduction approach.

The coordinate measuring machine (CMM) used for this work, Leitz PMM-C 700 [1], with a fixed bridge and a moving table, is an ultra-high precision metrology instrument with sub-micron accuracy. The non-contact Precitec LR optical probe [2], with $\sim 3 \mathrm{~nm}$ Z-resolution, is used to sample optical surfaces with improved precision over contact probes and reduce the risk of hardware damage. Tactile probes are also used to measure part's mechanical features that cannot be measured with the Precitec probe, due to access limitations. As an optical metrology tool, the CMM has the powerful and unique capability to provide simple, 3D surface points that can be manipulated to obtain the as-built prescription and surface error, including optical surface alignment (i.e., six degree of freedom pose). The CMM-measured, low-order aberrations are thus intrinsic to the surface and are not test- or misalignment-induced aberrations. These low order aberrations provide valuable information about the as-built prescription and the alignment of the optic with respect to the working frame, and hence should not be removed as often the case in an interferometric test. Furthermore, the CMM has large dynamic range --- measured in mm. The example shown in Figure 1 illustrates how an ultra-precision CMM rivals interferometry where the surface slopes are measurable and surpasses it when measuring surfaces with such large figure error that the interferometer fails.

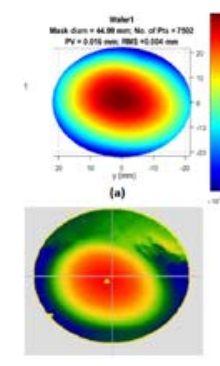

(e)

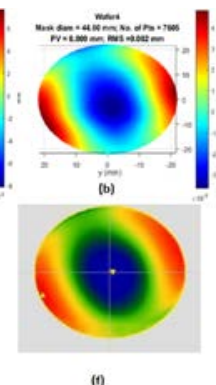

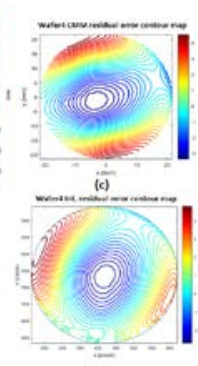

(s)

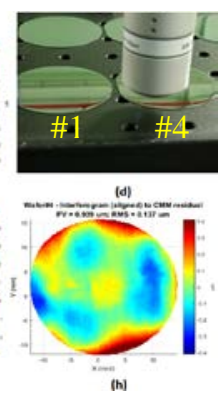

Fig. 1. CMM-Precitec scan vs. interferometric test of Silicon wafers with large surface figure error. (a,e) CMM \& int. measurement of wafer \#1, respectively; (b,f) CMM \& int. measurement of wafer \#4; (c,g) wafer \#4 residual error contour map, rotated, as measured by CMM \& int.; (d) photograph showing the Precitec measuring wafer \#4; (h) the residual error difference between CMM and the int. measurement. Note the loss of data around the edges of both wafers in the interferometric data sets. This contributes to the larger difference between the two metrologies reported in (h) around the edges of the wafer (i.e., those data are invalid). 
This summary is divided principally into two areas of metrology. The first focuses on utilizing CMM metrology for freeform optics and polynomial aspheric prescriptions as examples of challenging optics. The second discusses the alignment and testing of systems composed of these surfaces.

\section{Prescription retrieval}

CubeSat and other missions are getting more popular as the low-cost alternative to perform science for NASA, pushing optical designs to comply with the severe packaging constraints. Freeform surfaces can help reduce the system volume and even eliminate surfaces, in comparison to a more traditional design [3][4][5]. Since one can only fabricate what they can measure, advances in metrology are as important as advances in fabrication. This work will serve an independent verification method for these challenging components and will improve the industry's capability to perform acceptance testing for freeform components, in addition to aligning freeform systems for future flight applications.

Two XY polynomial, freeform mirrors for a compact telescope, "XY Penta," were successfully characterized, Fig. 2, and aligned using the CMM, Fig. 4. The prescriptions were chosen for a fictitious, U3 CubeSat-sized telescope that is well-corrected over a large field of view using two polynomial freeform surfaces with departure challenging enough to push fabrication and metrology technologies. The average percentage difference between the design and the as-built polynomial coefficients of M1 were found to be less than $2 \%$.

The L’Ralph instrument for the planetary science Lucy mission posed another system with mirror prescriptions that would be challenging to measure via traditional optical testing. L'Ralph is a three mirror anastigmat comprised of fast, polynomial aspheres that are all-aluminum and fabricated via diamond machining. We measured two of the system's engineering model (EM) mirrors, Fig. 3 [6].
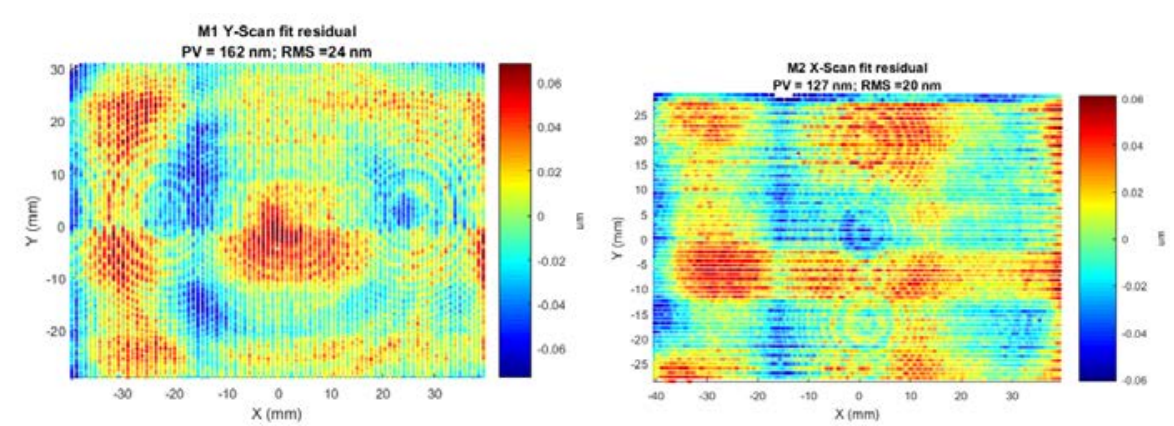

Fig. 2. CMM scan residual error maps of the XY Penta telescope primary, M1 (left), and secondary, M2 (right), calculated from the as-built prescription determined by multiple scans and many data fitting trials. Note the remarkable $\sim 20 \mathrm{~nm}$ RMS error for both mirrors, likely dominated by metrology artifacts (e.g., CMM and probe error, environmental instabilities).
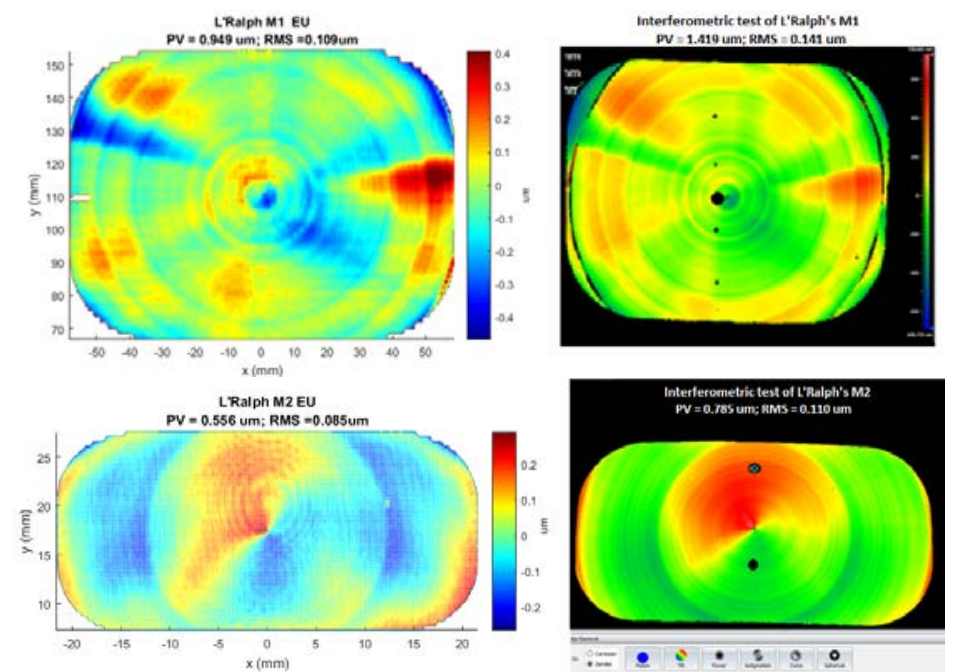

Fig. 3. CMM scan residual error maps of the L'Ralph M1 EM and the corresponding interferometric measurement (top), and CMM residual maps of L'Raph M2 EM and the corresponding interferometric measurement (bottom). The results appear to be dominated by tool marks from the diamond machining process. 


\section{Alignment and integration}

Optical surface alignment expressed as six degree of freedom (6 DoF) pose, three translations and three rotations with respect to the working coordinate system, is a byproduct of fitting the 3D surface data to the sag formula, to determine the residual error and the as-built surface prescription parameters. CMM metrology results for an optical component can guide a system's alignment by providing information on where the best-fit optical surface actually is relative to reference objects or fiducials, such as tooling balls (TBs), precision holes, marks, and/or highly flat planes. The optical prescription (i.e., optical surface parameters and pose, thicknesses, etc.) can also be updated using the CMM results.

This was the adopted approach to align the L'Ralph three-mirror-anastigmat based on the calibration of TB location for each mirror (Fig. 4). The system TBs were initially aligned to the CMM-calibrated positions, using a laser radar metrology instrument for system-level metrology. An interferometer was used to obtain a double-pass, end-to-end wavefront error measurement --- fringes were immediately obtained once the TBs were within $50 \mu \mathrm{m}$ from their calibrated positions. The final stages of alignment were guided by the optical model with feedback from interferometry and was performed in a record time for such a system. The optical ray trace model was then updated with the as-built surface prescriptions and alignment.

Indeed, one can also use the CMM to perform system-level metrology and alignment, potentially improving alignment uncertainty by better than a factor of 10 . To demonstrate this, we used the non-contact probe and a semiautomated routine to measure the location of TBs on the sides of each mirror for the XY Penta telescope, calculating the required 6 DoF transformation, adjusting a 6 DoF stage, and iterating the process until the system was aligned to the $\sim \mu \mathrm{m}$-level. We then verified alignment by performing an end-to-end interferometric test and comparing those wavefront error results to those predicted by the as-built optical model.

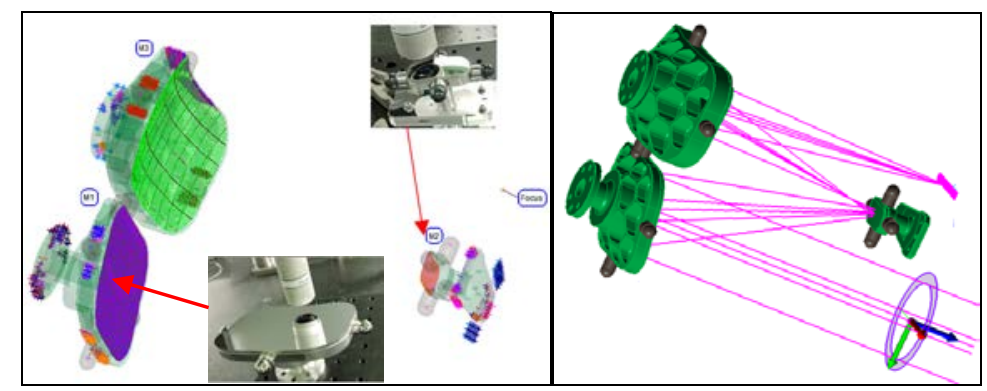

Fig. 4. a. View from metrology visualization software showing the three mirrors of L'Ralph system at 6 DoF poses informed by component-level, CMM metrology. Inset photographs show the non-contact probe measuring the optical surfaces of M1 and M2. b. The system layout is again shown from a different vantage point. Note the inserted renderings of mirror substrate designs from CAD.
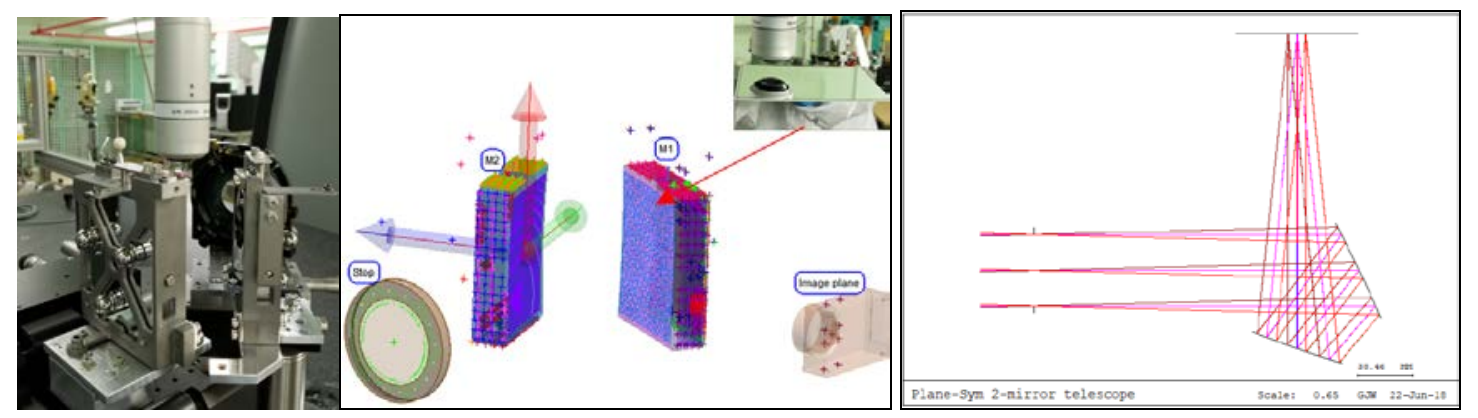

Fig. 5. a. Photograph showing the two-mirror XY Penta being aligned using the CMM-Precitec probe (left). b. View from metrology software showing measurements of all mirror features and surfaces, including the aperture stop and select field points on the focal surface at the detector. c. View of the optical system layout from the ray trace model.

\section{References}

[1] Hexagon Metrology, "Leitz PMM-C Line Data Sheet 2016-6_EN.pdf.”.

[2] Hexagon Metrology, "Leitz Precitec LR_brochure_en.pdf."

[3] O. Fähnle, D. W. Kim, R. Williamson, "Freeform Optics,” Opt. Eng., vol. 55, no. 7, p. 071201, 2016.

[4] G. J. West and J. M. Howard, “Application for Freeform Optics at NASA,” EPIC Freeform Workshop, 2019.

[5] J. M. Howard and G. West, "Freeform Optics at NASA," in Imaging and Applied Optics 2015, 2015, p. FW3B.1.

[6] NASA, "Lucy Spacecraft.” [Online]. Available: http://lucy.swri.edu/mission/Spacecraft.html. 